DOI: 10.12731/2227-930X-2020-1-35-49

УДК 629.13

\title{
АНАЛИЗ ОСТАТОЧНЫХ ДЕФОРМАЦИЙ В ЭЛЕМЕНТАХ КУЗОВА ЛЕГКОВОГО АВТОМОБИЛЯ МЕТОДОМ ПРЯМОГО ИНТЕГРИРОВАНИЯ
}

\author{
Аблаев P.P., Аблаев А.P., \\ Абрамова Л.С., Ксенофонтова В.А.
}

В настоящее время существует множество различных методов определения скорости движения транспортного средства в момент столкновения. В условиях ограниченных исходных данных для экспертов, таких как следь колес на проезжей части в условиях места происшествия, наиболее оптимальным методом является метод определения скорости автомобиля в момент столкновения, основанный на учете затрат кинетической энергии на деформацию элементов и деталей автомобилей. В связи с чем, статья посвящена проблеме анализа остаточных деформаций в элементах кузова автомобиля при определении скорости его движения в момент столкновения. Проведен анализ различных видов остаточных деформаций и методов их оченки. Предложен метод прямого интегрирования для анализа остаточных деформациий в элементах кузова легкового автомобиля, поскольку он наиболее точно отражает всю механику образования деформачий кузова и на уровне низкой трудоемкости. Этот фактор является одним из важных при выборе методики исследования обстоятельств дорожно-транспортного происшествия. Предложенная методика показывает наименьшую погрешность вычислений при определении скорости движения автомобилей в момент столкновения.

Цель - разработать и обосновать модель учета затрат кинетической энергии да деформацию конструктивных элементов кузова легкового автомобиля при определении его скорости движения в момент столкновения на основании анализ остаточных 
деформаций в элементах кузова автомобиля методом прямого интегрирования.

Метод или методология проведения работы: в статье использовались системный подход, методы анализа и математического моделирования.

Pезультаты: предложенная в работе методика дает возможность на стадии исследования обстоятельств дорожно-транспортного происшествия определить скорость движения автомобилей в момент столкновения по их деформациям, которая более полно учитывает все затраты кинетической энергии на механизм деформирования сложных кузовных элементов, имеющих ребра, гофры и другие элементы, повышающие их жесткость при изгибе.

Область применения результатов: полученные результаты целесообразно применять в экспертной практике исследования обстоятельств и механизма дорожно-транспортного происшествия при определении скорости движения автомобилей в момент столкновения.

Ключевые слова: энергия; деформачия; напряженное состояние; автомобиль; скорость движение; столкновение.

\title{
ANALYSIS OF RESIDUAL DEFORMATIONS IN CAR BODY ELEMENTS BY DIRECT INTEGRATION METHOD
}

\author{
Ablaev R.R., Ablaev A.R., \\ Abramova L.S., Ksenofontova V.A.
}

Currently, there are many different methods to determine the speed of a vehicle at the time of collision. Under the conditions of limited initial data for experts, such as wheel tracks on the roadway at the scene of an accident, the best method is to determine the speed of a car at the time of collision, based on the amount of kinetic energy spent for the deformation of elements and parts of cars. In this regard, the article is devoted to the problem of analysis of residual deformations in the 
elements of the car body when determining the speed of its movement at the moment of collision. The analysis of various types of residual deformations and methods of their estimation is carried out. The method of direct integration for the analysis of residual deformations in the elements of the car body is proposed, since it most accurately reflects the entire mechanics of the deformation process for the car body at the low level of labor intensity. This factor is one of the most important when choosing a method for investigating the circumstances of a road accident. The proposed method shows the smallest error of calculations when determining the speed of cars at the moment of collision.

The research purpose is to develop and justify a model for accounting for the cost of kinetic energy for the deformation of structural elements of the car body when determining its speed at the moment of collision based on the analysis of residual deformations in the elements of the car body by direct integration.

Methodology the article uses a systematic approach, methods of analysis and mathematical modeling.

Results: the proposed method allows at the stage of investigation of traffic accidents to determine the speed of the vehicle at the time of collision in their deformations, which more fully accounts for all costs kinetic energy for the deformation mechanism of complex body elements having ribs, corrugations, and other elements, which increase the bending stiffness.

Practical implications: the obtained results should be used in expert practice to study the circumstances and mechanism of a traffic accident when determining the speed of cars at the time of a collision.

Keywords: energy; strain; stress state; car; speed movement; collision.

\section{Постановка проблемы}

В последнее время наблюдается значительный рост эксплуатируемых транспортных средств в мире, что обуславливает повышение в геометрической прогрессии количества дорожно-транспортных происшествия. При этом, современные транспортные средства характеризуются высокими значениями средней скоро- 
сти эксплуатации, что оказывает влияние на повышение количества происшествий с тяжкими последствиями. Одним из способов профилактики наступления дорожно-транспортного происшествия является вынесение объективного наказания правонарушителям, что зачастую невозможно без объективного анализа обстоятельств дорожно-транспортного происшествия и установления фактических значений скоростей движения транспортных средств в момент столкновения $[1,2]$.

Существуют множество различных математических моделей, которые позволяют установить значение скорости движения транспортных средств в момент столкновения $[1,10,13]$, однако для их использования в экспертной практике необходимы дополнительные данные о следах, которые остались на месте происшествия и которые, к сожалению, не всегда корректно фиксируют в первичных документах. Этот факт исключает возможность использования классических методов исследования и требует непосредственной оценки и анализа остаточных деформаций кузовных элементов транспортных средств, при определении скорости движения этих автомобилей в момент столкновения.

Цель статьи - разработать и обосновать модель учета затрат кинетической энергии да деформацию конструктивных элементов кузова легкового автомобиля при определении его скорости движения в момент столкновения на основании анализ остаточных деформаций в элементах кузова автомобиля методом прямого интегрирования.

\section{Изложение основного материала}

Для обоснования косвенных методов анализа рассмотрим ряд задач для пластически деформируемых элементов конструкции легкового автомобиля, при этом в качестве основной меры деформации будем принимать энергию деформации или эквивалентную ей работу. Рассмотрим одномерный случай. Пусть элемент конструкции имеет первоначальную длину $l_{o}$ и площадь поперечного сечения $F_{o}$ (рисунок 1) [5]. 
Под действием усилия $P$ на $i$-м шаге деформации длина элемента уменьшится на величину $d l_{i}$, тогда приращение работы деформирования будет равно (1) [3]:

$$
d A_{\partial i}=P d l_{i}
$$

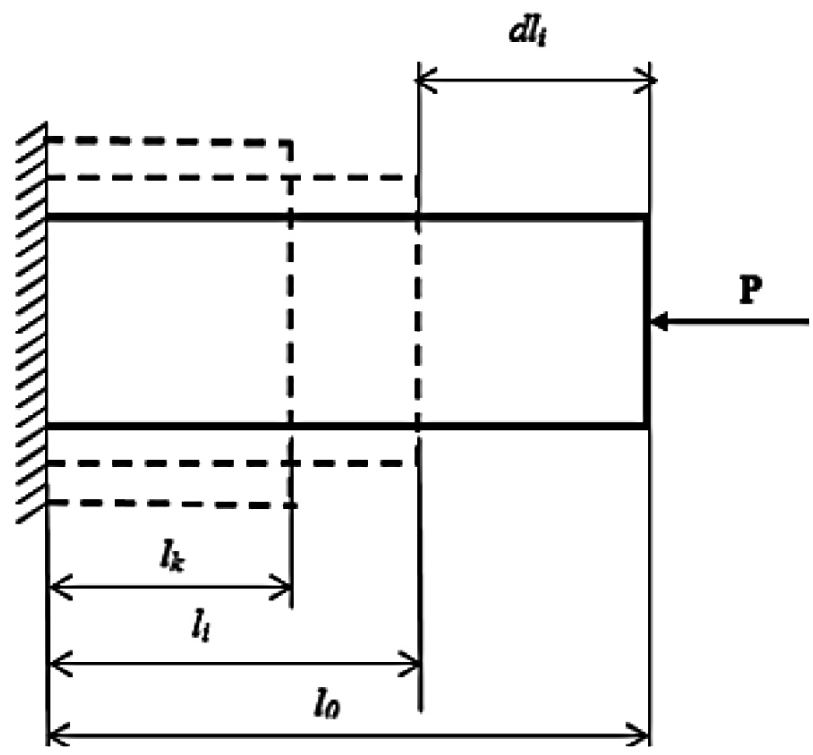

Рис. 1. Одномерная пластическая деформация элемента конструкции

В момент, когда элемент конструкции будет находиться в конечном состоянии $l_{k}$, полная работа деформирования будет равна (2):

$$
A_{\partial}=\int_{l_{0}}^{l} P d l
$$

С учетом знака работы поменяем пределы интегрирования (3):

$$
A_{\partial}=\int_{l}^{l_{0}} P d l
$$

Если усилие $P$ представить как распределенное $q_{c p}$ по площади $F_{0}$, а площадь $F_{0}$ выразить через объем $V_{0}(4)$

$$
F_{0}=\frac{V_{0}}{l_{0}} .
$$


Тогда интеграл (3) можно записать в виде (5):

$$
A_{\partial}=V_{0} q_{c p} \int_{l}^{l_{0}} \frac{d l}{l} \text {. }
$$

В выражении (5) объем $V_{0}$ вынесен за знак интеграла, так как при пластических деформациях объем тела не меняется [7, 12]. Среднее давление $q_{c p}$ на поверхности контакта также выносится за знак интеграла, так как согласно теореме о среднем в теории пластичности $[3,14]$, давление на поверхности пластически деформируемого тела принимается равным $q_{c p}$ с высокой степенью точности.

Выполняя интегрирование, получим (6):

$$
A_{\partial}=V_{0} \sigma_{s} \ln \frac{l_{0}}{l} \text {. }
$$

При больших деформациях среднее давление $q_{c p}$ достигает предела текучести и тогда работа деформирования в одномерном случае может быть определена с помощью выражения (7):

$$
A_{\partial}=\sigma_{s} V_{0} \ln \frac{l_{0}}{l},
$$

где $\sigma_{s}$ - напряжение текучести материала, при этом упругая составляющая энергии деформации составит $A_{y}=\frac{1}{2} q_{c p} \Delta l$.

Рассмотрим двумерный случай деформации. После прекращения действия активных сил, вызвавших пластическую деформацию в теле, форма тела будет изменена $[3,4,5,6]$. Характерный пример двумерной пластической деформации представлен на рисунке 2.

Под действием более жесткого тела I, в деформируемом теле II возникли пластические (остаточные) напряжения, поле распространения которых характеризуется линиями скольжения $\mathrm{O}^{\prime} \mathrm{C}, \mathrm{O}^{\prime} \mathrm{D}$ и так далее. Вдоль линий скольжения касательные напряжения достигают максимума и материал течет. Известно $[6,8,11,12]$, что вдавливание I в II вызывает распространение пластических деформаций на следующие расстояния (8):

$$
A C=B D=2 a \text {. }
$$

Линии O'C и O'D представляют собой полуокружности с радиусом $2 a$. Энергия деформации для этой схемы может быть вычислена по формуле (9): 


$$
\ni_{\partial}=A_{\partial}=2(\pi+2) \sigma_{S} a h,
$$

где $\sigma_{s}$ - напряжение текучести;

$h$ - глубина внедрения.

В случае, если деформирующее тело имеет не плоскую форму, а например, сферическую (рисунок 3), то работу деформации следует вычислять с помощью выражения $(10)[9,12]$ :

$$
A=\int_{h}^{h_{o}} P d h,
$$

где усилие $P$ вычисляется по формуле (11):

Для случая сферы (12):

$$
P=\iint \sigma_{H} d F \text {. }
$$

$$
\sigma_{H}=\sigma_{S}\left(1+\frac{\pi}{2}-\gamma\right) \text {. }
$$

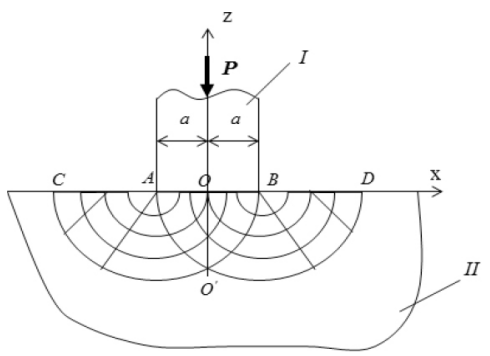

Рис. 2. К определению зоны пластической деформации в деформируемом твердом теле

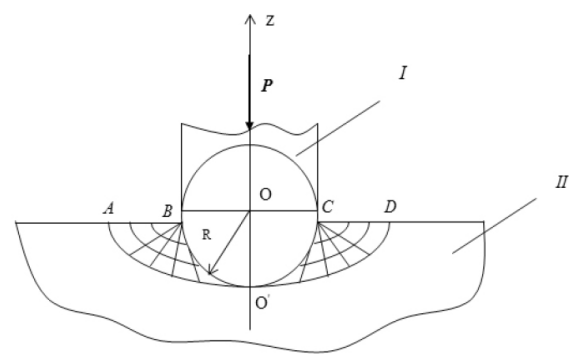

Рис. 3. Пластическое деформирование мягкой стали сферическим твердым телом

Большой интерес представляет задача анализа пластических деформаций при изгибе элементов конструкции из листовой стали, таких как внешние панели кузова, капот, крышка багажника, и др. Обозначим через $R_{l}$ и $R_{I I}$ радиусы внутренней и наружной поверхностей изгибаемого листа (рисунок 4).

В результате изгиба лист получил деформацию, характеризуемую углом изгиба $\varphi=\varphi_{0}+\Delta \varphi$, при этом радиусы точек внутренней и наружной поверхностей стали $r_{1}$ и $r_{2}$. Возникающие при этом пластические деформации зависят от угла $\varphi$ и могут быть определены по формуле (13): 


$$
\varepsilon_{t}=\frac{r_{1} \varphi-l_{0}}{l_{0}}
$$

где $l_{0}$ - ширина изгибаемого элемента.

При разгрузке изогнутого листа окончательный радиус срединной поверхности $r_{\text {ост }}$ определится из выражения (14)

$$
\frac{1}{r_{\text {ост }}}=\frac{1}{r_{0}}-\frac{1}{r_{\text {разгр }}},
$$

где $r_{\text {разг }}$ - радиус упругой разгрузки (15):

$$
\frac{1}{r_{\text {разгр }}}=\frac{M}{E J},
$$

где $M, E, J$ - характеристики упругого изгиба.

Внешние панели, изготовленные из листового материала в виде пластин, при действии сжимающей нагрузки (рисунок 5), обычно теряют устойчивость $[11,12,14]$.

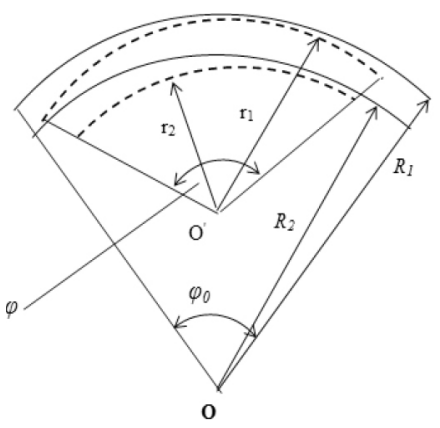

Рис. 4. Пластический изгиб стального листа

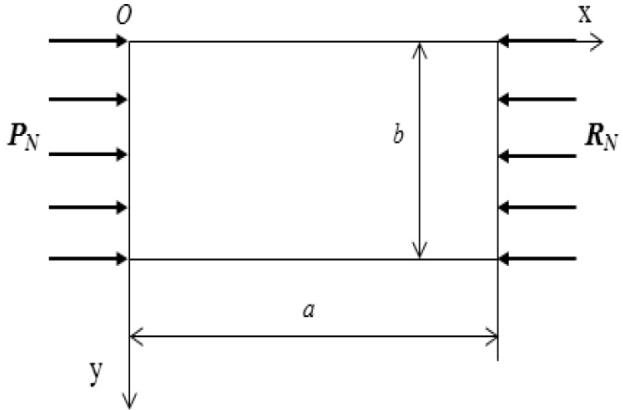

Рис. 5. К определению критической сжимающей силы для деталей типа пластин

Последующее деформирование не требует дополнительных затрат энергии и ими можно пренебречь. Действие сжимающей силы $P_{N}$ и равной ей реакции $R_{N}$ определяется из уравнения устойчивости (16):

$$
\nabla^{2} \nabla^{2} u_{z}=-\frac{P_{N}}{D} \frac{\partial^{2} u_{z}}{\partial x^{2}},
$$

где $u_{z}$ - прогиб пластины; 
$D=\frac{E h^{3}}{12\left(1-v^{2}\right)}-$ цилиндрическая жесткость;

$h$ - толщина пластины;

$E, v$ - характеристики материала.

Минимальное значение силы $P_{N}$, при которой пластина быстро изогнется, определяется интегрированием (17):

$$
P_{N}=\frac{\pi^{2} D\left(\frac{m^{2}}{a^{2}}+\frac{n^{2}}{b^{2}}\right)}{\frac{m^{2}}{a^{2}}} .
$$

Если деформированная плоская панель имеет более одной полуволны изгибной деформации, то в (17) следует определить $m$ из выражения (18):

$$
\mu=\sqrt{m^{2}+m}
$$

где $\mu=\sqrt{2}-$ при изгибе в виде двух полуволн, $\mu=\sqrt{6}-$ три полуволны и так далее.

Энергия, затраченная на деформацию такого элемента конструкции, будет равна работе силы $P_{N}(19)$ :

$$
\ni_{\partial}=A_{\partial}=\frac{1}{2} P_{N} \Delta x
$$

где $\Delta x=\arcsin \frac{m \pi x}{a}$.

Автомобильные панели пола и внешние обычно имеют ребра, гофры и другие элементы, повышающие их жесткость при изгибе. Такие элементы конструкции продолжают сопротивляться действию внешних сил и после потери устойчивости, при этом возникают большие деформации [9]. Для анализа таких случаев была рассмотрена задача деформации пластины произвольного переменного профиля (20):

$$
h=f(x, y) .
$$

Для учета возникающих больших деформаций были выведены модифицированные уравнения Кармана [9] (21):

$$
\left\{\begin{array}{l}
\nabla^{2} \nabla^{2} F=L_{1}(h, w, x, y) \\
\nabla^{2} \nabla^{2} W=L_{2}(h, F, w, x, y)
\end{array}\right.
$$


где $L_{1}$ и $L_{2}$ - дифференциальные операторы;

$F$ - функция напряжений;

$h$ - толщина пластины.

Решая эти модифицированные уравнения Кармана совместно, возможно получить уравнения для определения нормальных и касательных напряжений, действующих в срединной плоскости панели пола автомобиля.

\section{Выводы}

Предложенная в работе методика дает возможность на стадии исследования обстоятельств дорожно-транспортного происшествия определить скорость движения автомобилей в момент столкновения по их деформациям, которая более полно учитывает все затраты кинетической энергии на механизм деформирования сложных кузовных элементов, имеющих ребра, гофры и другие элементы, повышающие их жесткость при изгибе. Это дает возможность усовершенствовать математическую модель определения скорости движения автомобилей в момент столкновения по их остаточным повреждениям.

Перспективой дальнейших исследования является адаптация предложенных модифицированных уравнений Кармана в общую математическую модель определения скорости движения транспортного средства по его остаточным деформациям, предложенных в работе и практическая апробация усовершенствованной модели при исследовании дорожно-транспортного происшествия.

\section{Список литературы}

1. Аблаев Р.Р. Современные возможности исследования технической состоятельности данных, полученных в процессе следственного эксперимента при наездах на пешеходов / Р.Р. Аблаев, А.Р. Аблаев, В.А. Ксенофонтова // Современные технологии. Системный анализ. Моделирование. 2019. Т. 61, № 1. С. 91-97. DOI: 10.26731/1813-9108.2019.1(61). 91-97.

2. Аблаев Р.Р. Постановка задачи оптимальной ресурсоэффективной компоновки механизированных объектов / Р.Р. Аблаев, А.Р. Аблаев // 
Фундаментальные и прикладные проблемы техники и технологии. 2020. №1 (339). C. 70-74. DOI: 10.33979/2073-7408-2020-339-1-70-74.

3. Александров А.В. Основы теории упругости и пластичности / А.В. Александров, В.Д. Потапов. М: Высш. шк., 1990. 398 с.

4. Васидзу К. Вариационные методы в теории упругости и пластичности: Пер. с англ. М.: Мир, 1987. 542 с.

5. Ветрогон А.А. Уточнение количества энергии поглощенной кузовом автомобиля при ДТП / А.А. Ветрогон // Вестник СевНТУ №152, 2014. С. 134-136.

6. Гольчевский В.Ф. Экспертное исследование прочностных свойств кузовов транспортных средств, подвергшихся конструктивным изменениям: монография / В.Ф. Гольчевский, Н.Ю. Жигалов, Н.Ю. Гольчевская. Иркутск: ФГКОУ ВПО ВСИ МВД РФ, 2015. С. 56-69.

7. Falaleev A.P. Lifelong modelling of properties for materials with technological memory / A.P. Falaleev, V.V. Meshkov // AV IOP Conf. Series: Materials Science and Engineering. N 153 (2016).

8. Hiemer M., Barrho J. Observer design for road gradient estimation // Reports in Industrial Information Technology, Vol.7, Shaker Verlag, Aachen: pp. 23-30, 2004.

9. Кирсанов А.Р. Оптимизация прочности каркаса салона автомобиля при фронтальном ударе с использованием программного обеспечения решения задач линейной статики / А.Р. Кирсанов, С.К. Хализов, С.А. Курдюк, Е.О. Иванов, В.А. Овчинников // Вестник МГТУ им. Н.Э. Баумана. Сер. “Приборостроение”. Конструирование и технология. М.: МГТУ, 2005. №3. С. 119-126.

10. Ксенофонтова В.А. Методика определения скорости движения транспортного средства в момент столкновения по остаточным деформация элементов конструкции / В.А. Ксенофонтова, А.Р. Аблаев, Р.Р. Аблаев // Фундаментальные и прикладные проблемы техники и технологии. 2019. № 4-1 (336). C. 130-134. URL: https://www. elibrary.ru/item.asp?id=39217127 (дата обращения 08.04.2020).

11. Nurkhaliesa Balqis Hamzah, Halim Setan and Zulkepli Majid Reconstruction of traffic accident scene using close-range photogrammetry technique // Geoinformation Science Journal, Vol. 10, No. 1, 2010, pp. 17-37. 
12. Огородников В.А. Энергия. Деформации. Разрушение (задачи автотехнической экспертизы): монография / В.А. Огородников, В.Б. Киселев, И.О. Сивак. Винница, 2005. 204 с.

13. Совершенствование методов автотехнической экспертизы при дорожно-транспортных происшествиях [Текст]: монография / В.П. Волков, В.А. Ксенофонтова, В.Н. Торлин и др. Харьков: Изд-во ХНАДУ, 2010. 476 с.

14. Becker T., Reade M., and Scurlock B. Simulations of Pedestrian Impact Collisions with Virtual CRASH 3, Accident Reconstruction Journal, Vol. 26, No. 2, 2016, http://arxiv.org/abs/1512.00790.

15. Теребушко О.И. Основы теории упругости и пластичности / О.И. Теребушко. М.: Наука, 1984. 320 с.

\section{References}

1. Ablaev R.R., Ablaev A.R., Ksenofontova V.A. Sovremennye vozmozhnosti issledovaniya tekhnicheskoj sostoyatel'nosti dannyh, poluchennyh v processe sledstvennogo eksperimenta pri naezdah na peshekhodov [Modern possibilities of studying the technical consistency of data obtained in the course of an investigative experiment when hitting pedestrians]. Sovremennye tekhnologii. Sistemnyj analiz. Modelirovanie [Modern technology. System analysis. Modeling]. 2019. V. 61, № 1, pp. 91-97. DOI: 10.26731/1813-9108.2019.1(61). 91-97.

2. Ablaev R.R., Ablaev A.R. Postanovka zadachi optimal'noj resursoeffektivnoj komponovki mekhanizirovannyh ob"ektov [Setting the problem of optimal resource-efficient layout of mechanized objects]. Fundamental'nye i prikladnye problemy tekhniki i tekhnologii [Fundamental and applied problems of engineering and technology]. 2020. №1 (339), pp. 70-74. DOI: 10.33979/2073-7408-2020-339-1-70-74.

3. Aleksandrov A.V., Potapov V.D. Osnovy teorii uprugosti i plastichnosti [Fundamentals of the theory of elasticity and plasticity]. 1990. $398 \mathrm{p}$.

4. Vasidzu K. Variacionnye metody v teorii uprugosti i plastichnosti [Variational methods in the theory of elasticity and plasticity]. 1987. $542 \mathrm{p}$.

5. Vetrogon A.A. Utochnenie kolichestva energii pogloshchennoj kuzovom avtomobilya pri DTP [Specification of the amount of energy 
absorbed by the car body in an accident]. Vestnik SevNTU [Bulletin of SevNTU]. №152. 2014, pp. 134-136.

6. Gol'chevskij V.F., Zhigalov N.Yu., Gol'chevskaya N.Yu. Ekspertnoe issledovanie prochnostnyh svojstv kuzovov transportnyh sredstv, podvergshihsya konstruktivnym izmeneniyam [Expert study of the strength properties of vehicle bodies that have undergone structural changes]. 2015, pp. 56-69.

7. Falaleev A.P., Meshkov V.V. Lifelong modelling of properties for materials with technological memory. AV IOP Conf. Series: Materials Science and Engineering. No. 153 (2016).

8. Hiemer M., Barrho J. Observer design for road gradient estimation. Reports in Industrial Information Technology, Vol.7, Shaker Verlag, Aachen: pp. 23-30, 2004.

9. Kirsanov A.R., Halizov S.K., Kurdyuk S.A., Ivanov E.O., Ovchinnikov V.A. Optimizaciya prochnosti karkasa salona avtomobilya pri frontal'nom udare s ispol'zovaniem programmnogo obespecheniya resheniya zadach linejnoj statiki [Optimization of the strength of the car interior frame during a frontal impact using software for solving linear static problems]. Vestnik MGTU im. N.E. Baumana. Ser. "Priborostroenie". Konstruirovanie i tekhnologiya [Bulletin of the Bauman Moscow state technical University. Ser. "Instrument making”. Design and technology]. 2005. №3, pp. 119-126.

10.Ksenofontova V.A., Ablaev A.R., Ablaev R.R. Metodika opredeleniya skorosti dvizheniya transportnogo sredstva v moment stolknoveniya po ostatochnym deformaciya elementov konstrukcii [Method of determining the speed of a vehicle at the moment of collision by residual deformations of structural elements]. Fundamental'nye $i$ prikladnye problemy tekhniki $i$ tekhnologii [Fundamental and applied problems of engineering and technology]. 2019. № 4-1 (336), pp. 130-134.

11. Nurkhaliesa Balqis Hamzah, Halim Setan and Zulkepli Majid Reconstruction of traffic accident scene using close-range photogrammetry technique. Geoinformation Science Journal, Vol. 10, No. 1, 2010, pp. 17-37. 
12. Ogorodnikov V.A., Kiselev V.B., Sivak I.O. Energiya. Deformacii. Razrushenie (zadachi avtotekhnicheskoj ekspertizy) [Energy. Deformations. The destruction of (the problems of autotechnical expert appraisal)] 2005. $204 \mathrm{p}$.

13. Volkov V.P., Ksenofontova V.A., Torlin V.N. Sovershenstvovanie metodov avtotekhnicheskoj ekspertizy pri dorozhno-transportnyh proisshestviyah [Improving the methods of auto technical expertise in road accidents]. 2010. 476 p.

14. Becker T., Reade M., and Scurlock B. Simulations of Pedestrian Impact Collisions with Virtual CRASH 3, Accident Reconstruction Journal, Vol. 26, No. 2, 2016, http://arxiv.org/abs/1512.00790.

15. Terebushka O.I. Fundamentals of the theory of elasticity and plasticity. 1984. $320 \mathrm{p}$.

\section{ДАННЫЕ ОБ АВТОРАХ}

Аблаев Ремзи Рустемович, доцент кафедры «Экономика предприятия», кандидат экономических наук, судебный эксперт Севастопольский государственный университет ул. Университетская, 33, г. Севастополь, 299053, Российская Федеращия ablaev.expert@mail.ru

Аблаев Алим Рустемович, доцент кафедры «Энергоустановки морских судов и сооружений», кандидат технических наук Севастопольский государственный университет ул. Университетская, 33, г. Севастополь, 299053, Российская Федераиия alim_ablaev@mail.ru

Абрамова Лариса Сергеевна, доцент кафедры «Экономика предприятия», кандидат экономических наук Севастопольский государственный университет ул. Университетская, 33, г. Севастополь, 299053, Российская Федерачия ablaev.expert@mail.ru 
Ксенофонтова Виктория Анатольевна, доцент кафедры «Автомобильный транспорт», кандидат технических наук, судебный эксперт

Севастопольский государственный университет

ул. Университетская, 33, г. Севастополь, 299053, Российская Федерация

vaksenofontova.v@gmail.com

\section{DATA ABOUT THE AUTHORS}

Ablaev Remzi Rustemovich, associate Professor of the Department of enterprise Economics, Candidate of Economic Sciences, forensic expert

Sevastopol State University

33, Universitetskaya Str., Sevastopol, 299053, Russian Federation ablaev.expert@mail.ru

Ablaev Alim Rustemovich, associate Professor of the Department "power Installations of marine vessels and structures", Candidate of Technical Sciences

Sevastopol State University

33, Universitetskaya Str., Sevastopol, 299053, Russian Federation alim_ablaev@mail.ru

Abramova Larisa Sergeevna, associate Professor of the Department of enterprise Economics, Candidate of Economic Sciences Sevastopol State University 33, Universitetskaya Str., Sevastopol, 299053, Russian Federation ablaev.expert@mail.ru

Ksenofontova Viktoriya Anatolievna, associate Professor of the Department of Automobile transport, Candidate of Technical Sciences, forensic expert

Sevastopol State University

33, Universitetskaya Str., Sevastopol, 299053, Russian Federation vaksenofontova.v@gmail.com 